\title{
RIO: An Al based Virtual Assistant
}

\author{
Samruddhi S. Sawant \\ Department of Information \\ Technology \\ NBN Sinhgad Technical Institute \\ Campus \\ Pune, India
}

\author{
Abhinav A. Bapat \\ Department of Information \\ Technology \\ NBN Sinhgad Technical Institute \\ Campus \\ Pune, India
}

\author{
Komal K. Sheth \\ Department of Information \\ Technology \\ NBN Sinhgad Technical Institute \\ Campus \\ Pune, India
}

\author{
Swapnadip B. Kumbhar \\ Department of Information Technology \\ NBN Sinhgad Technical Institute Campus \\ Pune, India
}

\author{
Rahul M. Samant \\ Professor \\ Department of Information Technology \\ NBN Sinhgad Technical Institute Campus \\ Pune, India
}

\begin{abstract}
In this world of corporate companies, a lot of importance is being given to Human Resources. Human Capital Management (HCM) is an approach of Human Resource Management that connotes to viewing of employees as assets that can be invested in and managed to maximize business value. In this paper, we build a chatbot to manage all the functions of HRM namely -- core HR, Talent Management and Workforce management. A chatbot is a service, powered by rules and sometimes artificial intelligence that you interact with via chat interface. The service could be any number things, ranging from functional to fun, and it could live in any major chat product. The chatbot deployed in this project will be tailor-made according to the company. Some aspects will be handling FAQ's, getting notice periods according to designation etc.
\end{abstract}

\section{Keywords}

HCM, NLP, Microsoft bot framework.

\section{INTRODUCTION}

Organizations have realized that their real assets are the human capital available to them. The output achieved from them can make or break a company. A large amount of resources are dedicated to acquire them, train them and get maximum output from them. This has led to the development of the domain Human Capital Management (HCM). HCM generally deals with a few key features such as recruitment, onboarding, training (or at least training support and tracking) and performance management. The best HCM software can be seamlessly integrated with other HR software solutions and even third party applications like social media, accounting or payroll outsourcing. Increasingly, HCM software vendors are starting to offer features that were typically offered with HRIS and HRMS solutions, like time and attendance tracking. We intend to provide a solution for this domain by creating a chatbot. Chatbot's are taking the tech world by storm [14]. This technology - which helps humans converse with computers in their native language via a computer interface is gaining popularity in a variety of scenarios, especially customer service. Most of the businesses first heard about chatbot between the years 2015 and 2016. A survey conducted by Mind Bowser [16] found out that industries like Ecommerce (90\%), Insurance (80\%), Healthcare (75\%), Hospitality (57\%), and Recruitment $(30 \%)$ would be benefitted by such virtual assistants. The rise of messaging apps, the explosion of the app ecosystem, advancements in artificial intelligence (AI) and cognitive technologies, a fascination with conversational user interfaces and a wider reach of automation are all driving the chatbot trend. A chatbot can be deployed over various platforms namely Facebook messenger, Slack, Skype, Kik, etc. The most preferred platform among businesses seems to be Facebook messenger $(92 \%)$. There are around $80 \%$ of businesses that would like to host their chatbot on their own website. It is recommended creating chatbots that specialize in specific tasks, provide recommendations to users and excel at helping users complete those tasks. Effective chatbots will demonstrate an understanding of user needs [18] and complement these needs with quick access buttons and images that depict the options available. By incorporating these visual aids, chatbot can reduce the time and effort spent on interacting with the chatbot, resulting in a quality user experience.

\section{HISTORY}

1. German scientist Joseph Weizenbaum developed ELIZA in 1966 considered as the first chatbot. It behaved like a therapist by rephrasing the statements of user and posing them back as questions. Mimicked human conversation by matching use prompts to scripted responses-it was able, at least for a time, to pass the Turing artificial intelligence test [3], [10].

2. Psychiatrist Kenneth Colby developed PARRY in 1972attempted to simulate a person with paranoid schizophrenia. Parry was described as an Eliza with an attitude. It used a combination of assumptions, attributions and 'emotional responses' [3].

3. British programmer Rolo Carpenter developed JABBERWACKY in 1982. It was mostly used entertainment purposes. It attempted to move from text based to voice based [3].

4. Dr. Wallace developed ALICE (Artificial Linguistic Internet Computer Entity) an open source natural language AI based chatbot to form responses to queries. It forms responses based on some pattern matching. The basic unit of AIML is category. Each 
category consists of an input or question, an output or an answer and an optional context. The questions are called pattern. The answer is called the template. The two types of optional context are called "that" and "topic". The pattern matching while working with AIML consists only words, spaces and wildcard symbols _ and * [3].

5. In 2006 IBM came up with WATSON that used natural language processing and machine learning to reveal insights from large amounts of data. It was originally developed to compete in Jeopardy which it won in 2011 against two former winners. Watson uses IBM's deepQA software and Apache's UIMA framework [3].

6. In 2011 Apple came up with the popular Siri that used Automatic Speech Recognition that converted human voice commands to text. Siri played the role of a personal assistant. It used NLP to convert the converted text to parse text and then analyzed it to give out answers. When Siri recognized that the human has asked a question but it does not have an adequate answer to the question, it directed the query to WolframAlpha that performed general search operations [3].

7. In 2014, Microsoft released CORTANA for Windows 10. It was Microsoft's version of a personal assistant. Cortana gets its NLP capabilities from Tellme Networks and are coupled with a Semantic search database called Satori [3].

8. In 2015, Amazon released ALEXA. Alexa could stream podcasts, play audio books. It could also control smart devices using itself as a home automation hub. Alexa uses Amazon's own Amazon Comprehend for its natural language processing capabilities [3].

9. In 2016 Microsoft launched TAY based on Python, Java and Node.js but had to be taken down 16 hours into its launch because the chatbot started posting inflammatory content through its twitter handle and caused an uproar leaving Microsoft red faced [3].

10. In 2017 HDFC bank launched EVA, a chatbot that could get customers quick access to the bank's product details, fees and charges for various product details, application processes etc. EVA has served over 350,000 unique customers and has addressed over 2.7 million customer queries successfully. EVA uses NLP and AI like every modern chatbot.

\section{LITERATURE SURVEY}

We start with a paper that talk's about the basic design and implementation of a chatbot. However it failed to perform complex activities and queries [1], [11].

Next up a paper that compared the very first chatbot ELIZA and ALICE where the researcher ruled that it was easier to make bots using ALICE because of it was implemented using rule based pattern matching [2].

Next step was a study of different types of models of a chatbot from an article that compared them [13].

A chatbot is applicable in different domains. A few examples would be

Education domain - A virtual assistant is implemented for college students, solving college related queries. However the author suggested that neural networks could be a future implementation of the system and data encryption and data compression could be used to secure data [8].

Business domain - A chatbot could answer FAQs using Artificial Intelligence Markup Language (AIML) and Latent Semantic Analysis (LSA). The system would automatically route to LSA block if AIML failed to provide answer to a particular user query. Chappie [7] a semi-automatic chatbot converses with the user and finds out his intent. On learning the intent of the user it could transfer the user to associated expert. It achieved $65-67 \%$ accuracy using Naïve Bayes Classifier, SVM. Future scope was that Chappie could be scaled to handle the entire conversation.

Banking domain - A banking assistant that carries out banking tasks efficiently and in no time. This chatbot was built on the techniques of AIML and NLP but it failed to provide 3 tier architecture and its implementation on web services to support multiple clients [5], [6].

NLP is the basic technique on which a chatbot works. An article by J. Crawford spoke about what NLP is, why we need it and its basic implementation [12].

We reviewed how a chatbot was answering questions like how will AI powered customer service help customer support agents? How a chatbot assistant can help a business? How to design a conversation that could influence a person? What can be the cons of implementing a chatbot in HR domain? We've reviewed this from an article in chatbot magazine.

\section{PROPOSED WORK}

A survey was under taken at the HR Tech Conference and Expo [17] of more than 350 attendees. The following were the survey results:

Any information regarding leaves, company policies are made available when the employees demanded anytime, anywhere said $99 \%$ of the respondents.

$47 \%$ said that they would prefer to get information from such virtual assistant.

In the end $92 \%$ of the respondents agreed that the future Chabot's will be significant to help employees find information they need instantly.

The idea behind this chatbot is that it will give out information to a particular employee on a wide range of topics directly without having to consult the HR personally each time. It will focus on particular requirements of the company. Primary functionalities will include handling FAQ's, getting notice period according to the designation of that particular employee. The same survey also helped in understanding the most common employee's query like paid time off, open enrollment, maternity leave, leave of absence, etc. The following figure depicts the queries that the employees found comfortable asking a chatbot. 


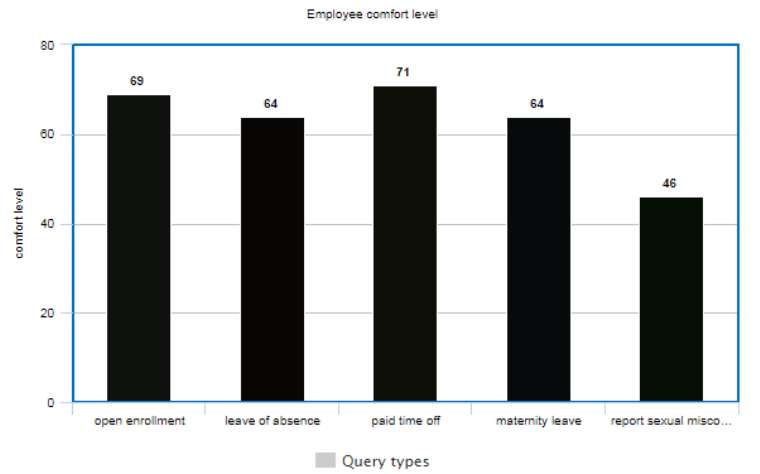

Fig.1 Common Employee Queries

Suppose an employee needs to talk to another employee on the intercom then it will provide an extension list. It will review an employee's performance by taking feedback from other employees and complete the appraisal cycle. Also employee can update his major life events. In short, most of the HR's work will be digitized and easy to access.

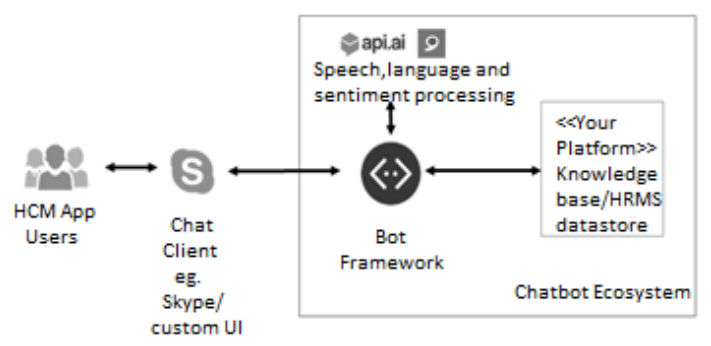

Fig 2: Architecture of Chatbot

\subsection{HCM App Users}

The users of this application are the employees of the company where this chatbot will be deployed.

\subsection{Chat client}

The user interface can be deployed over existing platform or a custom UI. Our UI will be a custom UI, in accordance with the company's specification.

\subsection{Chatbot Ecosystem}

A chatbot ecosystem is built on two major components-

1. HRMS Database

2. Technology Used

\subsubsection{HRMS Database}

A typical HRMS database will consist of the entire information of an employee. The database is huge. It contains information in every field. The database will have information on name, address, email-id, phone no, region, country, job title, salary, department name, joining date, extension list, notice period in accordance of a job title etc.

\subsubsection{Technology Used}

Technologies used in our project are

1. Bot framework

2. Node.js

3. Microsoft LUIS

\subsubsection{Bot Framework}

Microsoft bot framework consists of Bot builder, bot Connector, and bot directory. It also has an emulator where we can test the working of the bot. Bot builder SDKs for Node.Js, .Net are available to build the bot. If we want our bot to be more interactive, we can incorporate Microsoft cognitive services such as Language Understanding Intelligence Service. In Microsoft bot framework, the role of bot connector is very important. The responsibility of bot connector is to connect with the different channels some of which are Facebook, Skype, Slack, Web Chat etc. Once the bot is built, next job is to publish and deploy the bot to the Microsoft azure cloud platform. For that, it is mandatory to create the azure bot service in the azure cloud platform. Using the azure bot service, the bot is deployed to the cloud [15]. Mind Bowser survey [16] showed that $41 \%$ of businesses prefer Microsoft Bot Framework as a bot building platform. 


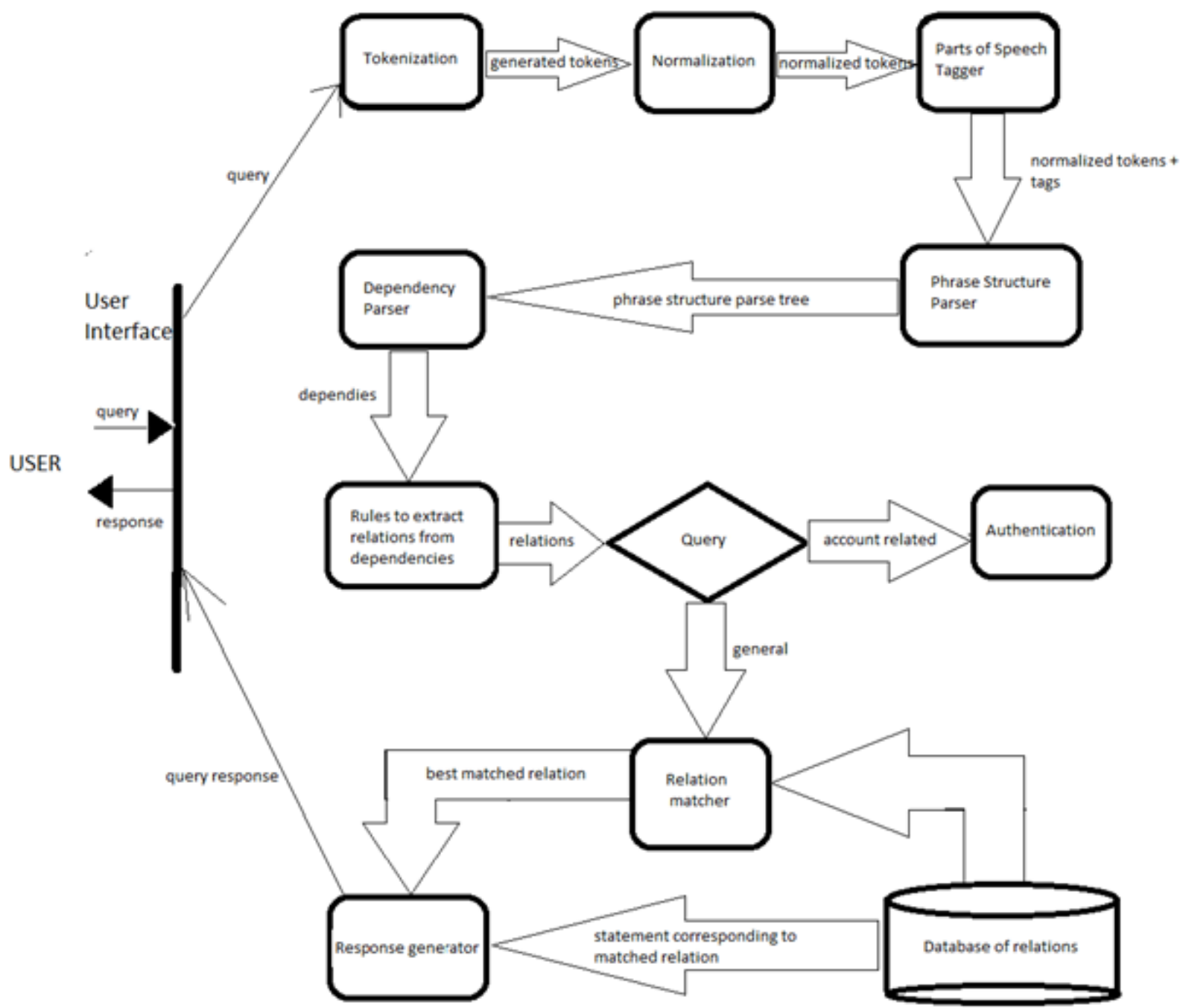

Fig 3. NLP Architecture

\subsubsection{Node.js}

Node.js is used to build and run a web application that uses JavaScript as its main language. It also has a built in web server and plug-ins that allow you to mold your application the way you want it to. Node.js allows language reuse that is, it requires you to learn only JavaScript on both client and server side. Node.js is lightweight. It allows a single thread per client request. It is the very foundation of non-blocking I/O a main feature of Node.js. It provides to object databases like Mongo DB. The advantage of JavaScript is that it's been around for a long time. So most editors and IDE's support it. Another advantage is that it can be hosted anywhere. Several web servers and cloud-based hosting providers support hosting of Node.js web applications out-of-the-box. Some examples would be Google, Microsoft Azure, and Amazon (AWS) etc. [9]

\subsubsection{Microsoft LUIS}

LUIS is what helps your application understand what you're saying when you say it in your own language. Thus LUIS allows developers to create an application that can understand what the user is saying in his natural language. It also allows the application to gather meaning from it. Basically LUIS allows a user program to enter a query in his natural language and get a response from the application. LUIS uses entities and intents. Applications are usually content related and centered on a topic that is domain specific. It allows the use of pre-built models from Cortana and Bing. Models deployment to a HTTP endpoint is very easy and it returns JavaScript Object Notation. Programmatic REST API's are available which helps the developer in automation of the application development process. LUIS offers free plans and is available in a number of languages like English, French, Italian, German, Spanish, Portuguese, Japanese, Brazilian, Chinese and Korean. A major component of LUIS is Natural Language Processing or NLP. The basic architecture of NLP can be drawn as shown in Figure 3. The basic functioning of NLP can be summarized in the following way. The user will type in his query, in his natural language. A friendly user interface will accept this query and pass it on to the tokenizer. The tokenizer converts the query into tokens by using delimiters like commas or semicolons. The output of the tokenizer is given to a normalizer which will do pre-processing. It includes spell check, expanding acronyms and abbreviations. These normalized tokens are tagged, giving it some meaningful labels. The labeled output is given to a Phase Structure Parser which checks whether language follows a pre- defined syntax. This can be difficult. Thus some rules 
will be used and using probability we can find the best parse of the sentence. The grammar must be of the typer Probabilistic Context Free Grammar. Thus parse tree will be generated. This is further given to a dependency parser which will convert the Phase Structure rules into dependencies. Stanford's dependency parser is preferred. The out of this parser will give us the dependencies between the words of the sentence. These dependencies are then used to extract some relations. This helps understand the context of the statements. The query is then authenticated. For e.g., in a banking assistant, it will check whether a user is allowed to access the details of his account. If it gets the go ahead then the relations are matched with existing relations in the database. The relations have a set of associated statements. The statements of the best matched relations are given to the response generator which gives it as query response to the user interface. The UI displays the statements as outputs to the clients query.

\section{RESULTS}

In this paper we have implemented a chatbot by the name RIO that carries out HR functionalities namely

\subsection{Getting the Extension list}

When an employee wants to contact another employee, he can simply ask the chatbot for the extension number of said employee, by typing his name. In companies having 1000s of employees working at one location this is especially useful since the employee have to ask around for the said employee's extension number.

\subsection{Getting Notice period}

Getting Notice period according to the employee's designation. Every company has different rules for notice periods. They're usually more stringent at fresher level and are relaxed up the order. The chatbot will tell the employee how much of a notice period he must serve.

\subsection{3 Finding the appraisal cycle}

All employees get an appraisal every year. The percentage depends on his performance through the year. The factors can be molded according to the policy and the user can know what percentage of appraisal he can get.

\subsection{Inserting/Updating life events}

Most companies have policies that will look after their employees. Along with that with that there are always security concerns. So most companies will demand complete information from their employees, so that it can be accessed whenever needed. Common life event can be getting married, divorces etc.

\subsection{Handling FAQs}

The most common task the HR has to accomplish will be answering questions that the employees have about company policy. In most cases, these questions are very common or in some cases, an employee might find it awkward to approach the HR. He can simply ask the chatbot for the answer. If the chatbot has no answer, the chatbot will send the query to HR.

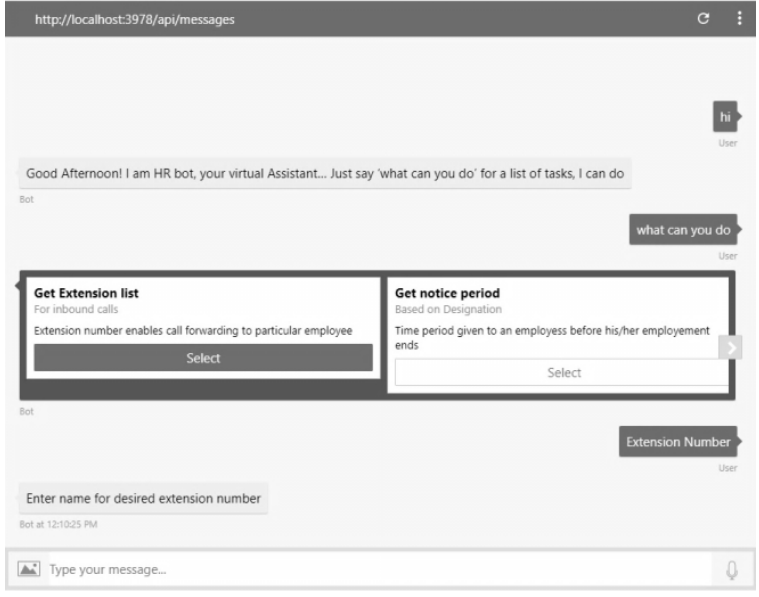

Fig 4: The Bot Laying Out the Users Options

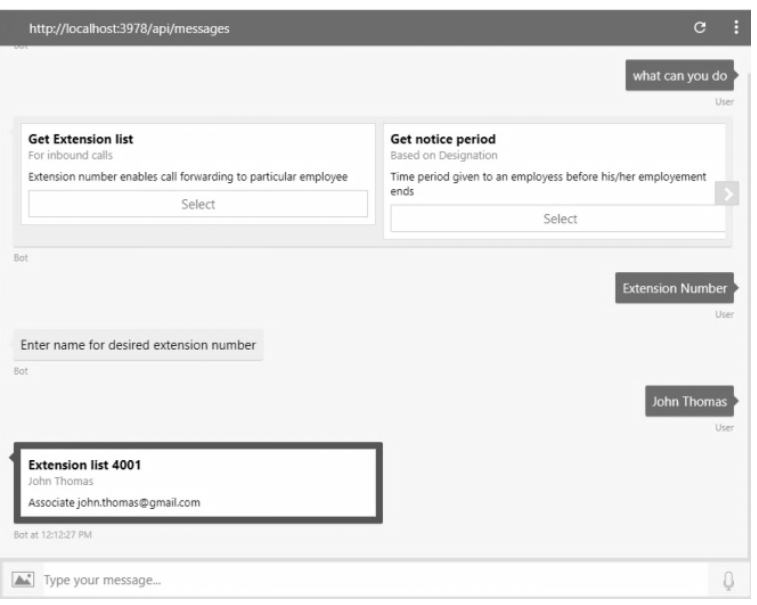

Fig 5. Getting extension no. after entering the required name

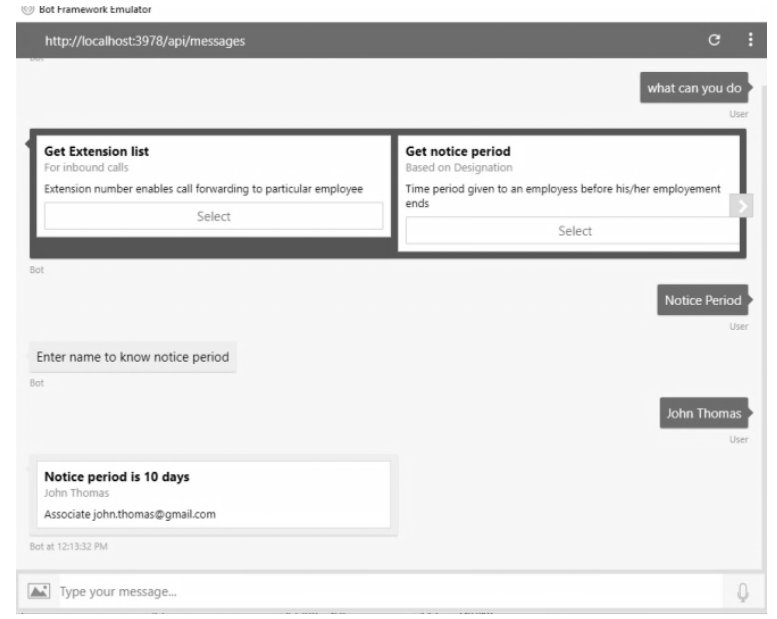

Fig 6. Getting the notice period you must see out

\section{CONCLUSION}

As of 2016 Facebook messenger had 33,000 chatbot. That number is now up to a 100,000 . It is evident that chatbot are future. Chatbots help in answering queries at any time and any place. Thus HR leaders are beginning to pioneer the use of AI into HR. A recent survey of $350 \mathrm{HR}$ leaders found that $92 \%$ leaders believe that the future of an enhanced service lies in Chatbot. The HR department has to deal with numerous queries on a daily basis. Chatbot can answer most of those 
queries. There lies the problem. Even a highly trained chatbot can fail to answer a query that is extremely unique query. So the chatbot will have to refer it to the HR personnel. But that'll be a unique case. The pros outweigh the cons. This chatbot can help the company save millions. Even though the chatbot will not render the human obsolete, but a significant amount of work load will come down for the HR. He can focus on the important parts thus improving his more output.

\section{FUTURE SCOPE}

A chatbot can be made friendlier by making it multi-lingual, e.g. Hinglish (Hindi + English). This can be done using a combination Natural Language Understanding and Natural Language Generation. NLU is a technique that helps in converting unstructured inputs into structured form. NLG helps in enhancing the quality of response. Conversational Interface $(\mathrm{CI})$ a new approach to enhance the user experience by addition of UI elements like message menus, images, buttons etc. A chatbot can be made friendlier by incorporating voice recognition. We have used a text based in this chatbot because according to consumer surveys most people prefer a text based chatbot.

\section{ACKNOWLEDGEMENT}

This research was supported by Harbinger Systems. We thank Jino Thomas (Software Engineer) and Sameer Nair (Software Engineer) for assistance with Node js, Microsoft Bot Framework. We thank our college professors from NBN Sinhgad College of Engineering who provided insight and expertise that greatly assisted the research.

\section{REFERENCES}

[1] M. Dahiya," A Tool of Conversation: Chatbot", International Journal of Computer Sciences and Engineering E-ISSN: 2347-2693, Volume-5, Issue-5, May 2017.

[2] V. Sharma, M. Goyal, D. Malik, "An Intelligent Behaviour Shown by Chatbot System", International Journal of New Technology and Research (IJNTR) ISSN: 2454-4116, Volume-3, Issue-4, April 2017.

[3] A. Deshpande, A. Shahane, D. Gadre,M. Deshpande, Prof. Dr. P. M. Joshi, "A Survey of various chatbot implementation technique", International Journal of Computer Engineering and Applications ISSN 23213469, Volume XI, Special Issue, May 17.

[4] A. Patil, K. Marimuthu, N. Rao A, R. Niranchana, "Comparative study of cloud platforms to develop a Chatbot", International Journal of Engineering \& Technology, Volume-6, Issue-3, 2017.

[5] K. B. Shah, M. S. Shetty, D. P. Shah, R. Pamnani, "Approaches towards Building a Banking Assistant", International Journal of Computer Applications (0975 8887), Volume 166 - No.11, May 2017.

[6] Thomas N T, "An E-business Chatbot using AIML and LSA", Intl. Conference on Advances in Computing,
Communications and Informatics (ICACCI), Sept. 2016.

[7] B. Behera, "Chappie-A Semi-automatic Intelligent Chatbot".

[8] V.Y. Shetty, N.U. Polekar, S.U. Das, S. Pansambal, "Artificially Intelligent College Oriented Virtual Assistant", International Journal of Technical Research and Applications e-ISSN: 2320-8163, Volume-4, Issue-2, April 2016.

[9] S. Tilkov, S. Vinoski, "Node.js: Using JavaScript to Build High-Performance Network Programs", IEEE Computer Society, 2010.

[10] J. Weizenbaum, "Computational Linguistics", Communications of the ACM, Volume 9, January 1966.

[11] M. Schlicht, "The Complete Beginner's Guide To Chatbots - Chatbots Magazine," Chatbots Magazine, 20Apr-2016. [Online]. Available: https://chatbotsmagazine.com/the-complete-beginner-sguide-to-chatbots-8280b7b906ca. [Accessed: 06-Mar2018].

[12] J. Crawford, "Understanding the Need for NLP in Your Chatbot - Chatbots Magazine," Chatbots Magazine, 15Feb-2018. [Online]. Available: https://chatbotsmagazine.com/understanding-the-needfor-nlp-in-your-chatbot-78ef2651de84. [Accessed: 06Mar-2018].

[13] Pavel Surmenok. [Online]. Available: http://pavel.surmenok.com/2016/09/11/chatbotarchitecture/. [Accessed: 06-Mar-2018].

[14] "Artificial Intelligence - Chatbots Magazine," Chatbots Magazine. [Online]. Available: https://chatbotsmagazine.com/tagged/artificialintelligence. [Accessed: 06-Mar-2018].

[15] RobStand, "Bot Service Documentation - Tutorials, API Reference - Bot Service," Tutorials, API Reference - Bot Service | Microsoft Docs. [Online]. Available: https://docs.microsoft.com/en-us/bot-framework/. [Accessed: 06-Mar-2018].

[16] D.Robinson, A.Ghodake, P.Smith, G.Miller, W.Sims, G.Martin, "Chatbot Survey 2017-Current state of Chatbots and their outlook in 2017" Chatbots Journal [Online]. Available: http://mindbowser.com/chatbotmarket-survey-2017/. [Accessed: 06-Mar-2018].

[17] D.R.Bharadwaj, "Chatbots and the Future of Employees Experience" ServiceNow [Online]. Available: https://servicematters.servicenow.com/2017/11/09/chatb ots-and-the-future-of-employee-experience/. [Accessed: 06-Mar-2018].

[18] A. R. Gadiyar, "The Chatbot Imperative: Intelligence, Personalization and Utilitarian Design", Jan-2017. [Pdf], pp.2-9. Available at: http://www.cognizant.com [Accessed: 06-Mar-2018] 